\title{
Iterative Kriging-based Methods for Expensive Black-Box Models
}

\author{
Siyang Deng, Reda El Bechari, Stéphane Brisset, and Stéphane Clénet
}

Univ. Lille, Centrale Lille, Arts et Metiers ParisTech, HEI, EA 2697 - L2EP - Laboratoire d'Electrotechnique et d'Electronique de puissance, F - 59000 Lille, France

\begin{abstract}
Reliability-Based Design Optimization (RBDO) in electromagnetic field problems requires the calculation of probability of failure leading to a huge computational cost in the case of expensive models. Three different RBDO approaches using kriging surrogate model are proposed to overcome this difficulty by introducing an approximation of the objective function and constraints. These methods use different infill sampling criteria (ISC) to add samples in the process of optimization or/and in the reliability analysis. Several enrichment criteria and strategies are compared in terms of number of evaluations and accuracy of the solution.
\end{abstract}

Index Terms - Infill sampling criteria, kriging model, reliability analysis, reliability-based design optimization.

\section{INTRODUCTION}

$\mathrm{R}$ ELIABILITY-BASED DESIGN OPTIMIZATION (RBDO) approaches can be divided into Double-Loop (DLM), Single-Loop (SLM) and Sequential Decoupled Methods (SDM). They have emerged in the past few decades and become more and more popular in electromagnetics owing to their ability to account for uncertain parameters. However, for expensive black-box models, the computational burden can become unbearable.

To overcome this issue, iterative kriging surrogate models have been proposed to reduce the number of evaluations [1][3]. Infill Sampling Criterion (ISC) was used with the aim of improving the quality of the surrogate model, and searching for the solution of the optimization problem. However, the metamodel is established before starting RBDO and no enrichment is made during neither optimization nor reliability analysis.

With the purpose of enhancing the efficiency, different strategies including the choice of the ISC and the positioning of sample enrichments in the optimization process are investigated in this paper for each aforementioned type of RBDO approaches, so that the reliabilities are also analyzed by metamodels. A mathematical example is used to compare with classic RBDO, i.e. without kriging model, and highlight the most effective strategy. Then, RBDO of a transformer modelled by a time consuming model based on the Finite Element method is performed with the most effective strategy.

\section{INFILL SAMPLING CRITERIA}

Iterative surrogate-based optimization methods start with a small set of initial sampling points to create a preliminary metamodel. Then, the infill sampling criteria are considered as new objective functions to add points into the sample set and update the meta-model until the predicted error is less than a chosen tolerance. A great advantage of this approach is that it enhances the accuracy of meta-model and search the probabilistic optimum simultaneously with a small amount of samples.

Manuscript received June, 28 2017; revised September 1, 2017; accepted. Date of publication; date of current version. Corresponding author: Siyang Deng (e-mail: siyang.deng@centralelille.fr).
Expected Improvement (EI) criterion [4] is widely used for surrogate-based optimizations without constraints.

$$
E I_{f}=\left\{\begin{array}{cc}
\left(f_{\min }-\hat{f}\right) \Phi(z)+\hat{s}_{f} \phi(z) & \text { if } \hat{s}_{f}>0 \\
0 & \text { if } \hat{s}_{f}=0
\end{array}\right.
$$

where $f_{\min }$ is the best current sampled objective function value, $\hat{f}$ and $\hat{s}_{f}$ are the predicted value and the mean square error (MSE), $\phi(\cdot)$ and $\Phi(\cdot)$ denote the probability density function and the cumulative distribution function of the standard normal distribution respectively, and $z=$ $\left(f_{\min }-\hat{f}\right) / \hat{s}_{f}$.

However, as EI is multimodal, more attention should be paid on the infill criterion to be sure to find the global solution. The Weighted EI (WEI) criterion [5] seems to be more suitable as it adds weights into EI expression to balance exploration (right part) and intensification (left part).

$$
\mathrm{W} E I_{f}=\left\{\begin{array}{cc}
\omega\left(f_{\min }-\hat{f}\right) \Phi(z)+(1-\omega) \hat{s}_{f} \phi(z) & \text { if } \hat{s}_{f}>0 \\
0 & \text { if } \hat{s}_{f}=0
\end{array}\right.
$$

Choosing small weight $\omega$ prevents WEI from converging to a local minimum if the initial sampling is inside the security domain. This condition is quite difficult to satisfy for many devices, as their security domains may be small and sometimes discontinuous. To avoid this issue, a Modified WEI (MWEI) combined with the surrogate objective function is proposed:

$$
\mathrm{MWEI} I_{f}=\mathrm{WEI} I_{f}-\omega \hat{f}
$$

Investigations on the same example as in [5] show that a weight equal to 0.1 provide to a global optimum with less iterations.

For constrained problems, an extended method consists in multiplying the value of EI by the probability of feasibility (PF) [6]. However, PF may prevent the sampling on the constraint boundary where the deterministic optimum may lie. Another constraint handling method is the Expected Violation (EV) method [7] but the number of candidate points to evaluate can be very large. An alternative method is to use the predicted value of the constraint functions $\hat{g}$ directly as constraints in the infill sub-problem [8]. 


\section{INFILL STRATEGIES FOR RBDO METHODS}

RBDO is a combination of deterministic constrained optimizations and reliability analysis. For the first one, the design variables $d$ are the mean value of random variables $X$ and the standard deviations $\sigma$ are constant. For reliability analysis, $d$ and $\sigma$ are constants and the design variable $x$ is a realization of $X . \beta_{t}$ is the given target reliability index.

\section{A. Double-Loop Method}

DLM like Performance Measure Approach (PMA) [9] has a nested structure: The outer loop seeks for the optimum and the inner loop searches the Most Performance Target Point (MPTP) that maximize the constraint subject to a given reliability index.

There are two places where ISC can be introduced to improve the accuracy of kriging model: Outer loop and inner loop. For the inner loop, EI of $g$ is used to find MPTP by solving the optimization problem in Eq. (4)-(5) as the constraint on the reliability index is an explicit function of the design variables:

$$
\begin{gathered}
x^{*}=\underset{x}{\operatorname{argmax}} E I_{g}(x) \\
\text { s.t. }\|(x-d) / \sigma\|=\beta_{t} \\
E I_{g}=\left\{\begin{array}{cc}
\left(\hat{g}-g_{\max }\right) \Phi\left(z_{g}\right)+\hat{s}_{g} \phi\left(z_{g}\right) & \text { if } \hat{s}_{g}>0 \\
0 & \text { if } \hat{s}_{g}=0
\end{array}\right.
\end{gathered}
$$

where $z_{g}=\left(\hat{g}-g_{\max }\right) / \hat{s}_{g}, \hat{s}_{g}$ is the MSE of the constraint, and $g_{\text {max }}$ is the maximum sampled constraint value.

For outer loop, the criterion MWEI is preferred to avoid local solutions and the implicit inequality constraints are computed by the inner loop. However, as the two loops are nested, the enrichment in inner loop may bring out thousands of model evaluations. To test it, two strategies are proposed: the first one (PMA1) adds new samples only inside the outer loop, whereas the second (PMA2) enriches inside both loops.

\section{B. Single-Loop Method}

For SLM like Single Loop Approach (SLA) [10], the main point is that the inner loop optimization is replaced by an approximation based on a first order Taylor expansion to avoid the numerous evaluations required to find the MPTP.

It is important to note that due to its approximation, the method itself has already loose some precision. Therefore, it is expected that with a surrogate model, the two approximation errors will be added and the accuracy will be further reduced.

\section{Sequential Decoupled Method}

SDM like Sequential Optimization and Reliability Assessment (SORA) [11] are based on a series of sequential deterministic optimizations and reliability assessments. The main point is to shift the boundaries of constraints inside the feasible domain based on the reliability information obtained in the former iteration. The first optimization aims at searching the global deterministic optimum. Reliability assessment is then conducted to locate the MPTP corresponding to the target reliability index. Finally, new optimizations are carried out by taking into account the shift $t$ computed with MPTP.

Three strategies are proposed. In the first one (SORA1), the reliability analysis is the same as in the inner loop of PMA and

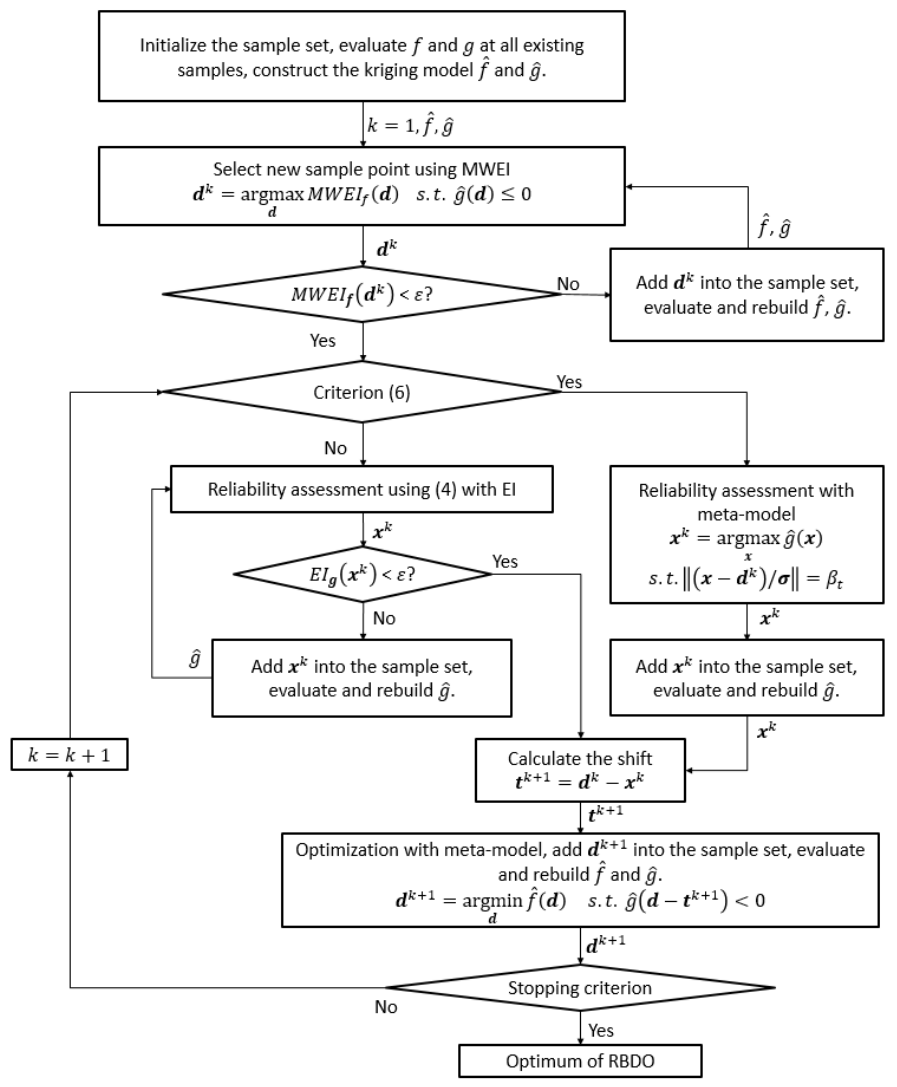

Fig. 1. The process of SORA3 strategy.

enrichment is made with EI criterion. For the deterministic optimizations, the constraints are computed with the metamodel $\hat{g}(d-t)$ and the MWEI is preferred in order to find the global solution.

The second strategy (SORA2) differs from the first one by the fact that enrichment of the kriging models with MWEI criterion is made at first iteration only. For all other iterations, the deterministic optimization is made with the meta-models $\hat{f}$ and $\hat{g}$.

For the third strategy (SORA3), if the deterministic optimum found in $k$-th cycle $d^{k}$ is close to any optimum of other $k-1$ cycles, as the former reliability assessments have already added points in this region, the accuracy is considered to meet the requirement so there is no need to add samples any more. The proximity criterion defined in (6) is checked before entering reliability analysis:

$$
\left\|\left(d^{k}-d^{i}\right) / \sigma\right\|<\beta_{t}, \quad i=1, \ldots, k-1
$$

where $d^{i}$ is the deterministic optimum found by the $i$-th cycle.

If (6) is satisfied, the meta-model of constraints is used directly and only MPTP are evaluated. For the deterministic optimizations, it takes the same strategy as SORA2. The flowchart of SORA3 is shown in Fig. 1 where $x^{k}$ is the MPTP of $k$-th cycle.

\section{COMPARISON OF STRATEGIES}

To assess the efficiency of kriging-based RBDO methods, the mathematical example in [12] with two variables and three constraints is analyzed. Noting that the random variables are 
Gaussian and their standard deviations are all equal to 0.3. Lower and upper bounds are 0 and 10 respectively for both variables. The target reliability index $\beta_{t}$ is chosen equal to 2 , so that the target probability of failure $P_{t}=\Phi\left(-\beta_{t}\right)=2.28 \%$.

TABLE 1

RESUlts OF MATHEMATICAL EXAMPLE USING DifFERENT STRATEGIES

\begin{tabular}{ccccc}
\hline \hline \multirow{2}{*}{ Strategy } & $\begin{array}{c}\text { Number of } \\
\text { evaluations }\end{array}$ & \multirow{2}{*}{ Optimal solution } & \multicolumn{2}{c}{ Optimal Maximal } \\
value & $P_{f}(\%)$ \\
\hline \hline SLA (exact model) & 165 & {$[2.2512 ; 1.9677]$} & -1.9953 & 2.32 \\
PMA/SORA & $3183 / 531$ & {$[2.2513 ; 1.9691]$} & -1.9945 & 2.27 \\
(exact model) & 26 & {$[2.2466 ; 1.9617]$} & -1.9996 & 2.59 \\
SLA & 29 & {$[2.2494 ; 1.9649]$} & -1.9972 & 2.44 \\
PMA1 & 1804 & {$[2.2513 ; 1.9691]$} & -1.9945 & 2.27 \\
PMA2 & $142 / 97 / 45$ & {$[2.2513 ; 1.9691]$} & -1.9945 & 2.27 \\
SORA1/2/3
\end{tabular}
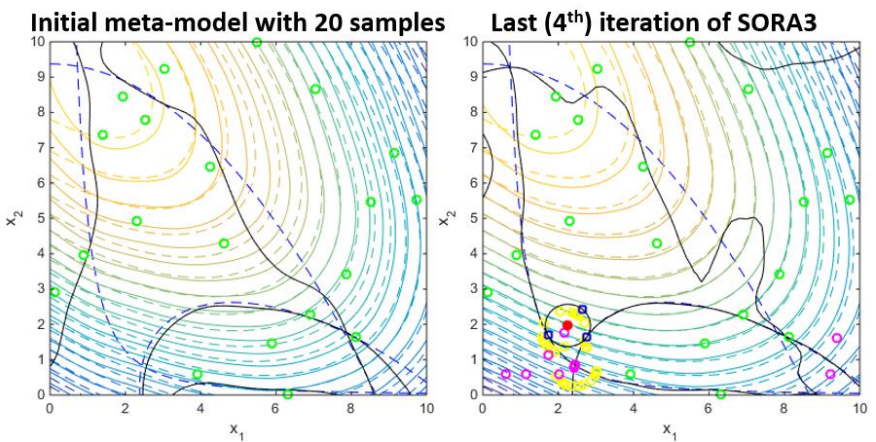

Fig. 2. Iterations of SORA3 for the mathematical example (green points are initial sampling, pink points are enrichment samples during deterministic optimizations, yellow ones are added by reliability analysis and blue ones are MPTPs at the current iteration, dashed lines and contours present the real constraints and objectives respectively while solid ones present the metamodels).

The results are given in Table 1 with an initial sampling of 20 points. The probability of failure $P_{f}$ is calculated by MonteCarlo Simulation (MCS) with $10^{6}$ samples. For comparison purpose, results given by classic RBDO methods without kriging are also presented.

All the iterative kriging-based RBDO methods lead to a reduced number of evaluations. SLA with kriging has the minimum number of evaluations but is not accurate enough as the maximum probability of failure is much greater than $P_{t}$ due to the approximation used to simplify the reliability analysis. As expected, PMA with infill during inner loops requires thousands of samples to evaluate. The other PMA strategy is faster but the accuracy is not sufficient. Kriging-based SORA strategies lead to the best result and the third one SORA3 is the most efficient. Fig. 2 shows two iterations of SORA3.

As SORA3 seems to be the most efficient meta-model strategy on this mathematical example, it is tested on the RBDO of a transformer with FEM.

\section{Electromagnetic Device}

The electromagnetic device is a single-phase safety isolating transformer with grain-oriented E-I laminations designed for installation in electric cabinet [13]. The primary and secondary windings are wound around the frame surrounding the central core (Fig. 3, left).

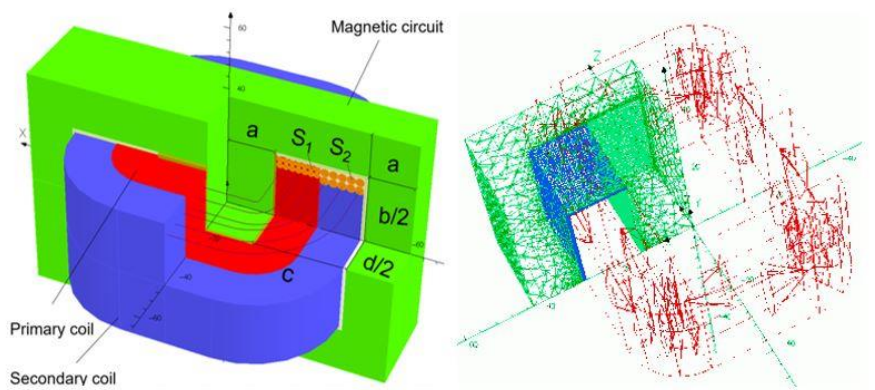

Fig. 3. Design variables of transformer and mesh.

\section{A. Finite Element Models}

Thermal and magnetic phenomena are modeled by using 3D FEA on the eighth of transformer due to symmetries. There are about 43,000 nodes and 290,000 edges in the model. The right part of Fig. 3 shows the mesh in the magnetic circuit, the insulating, the air gap, the frame and the opposing direction of currents in the primary and secondary windings that create flux in the gap between the coils (leakage flux).

For the electromagnetic modeling, all magnetic and electric quantities are assumed sinusoidal. Full-load and no-load simulations are used to compute all the characteristics. The iron losses are computed with the Steinmetz formula and the leakage inductances are calculated with the magnetic co-energy. The core magnetic nonlinearity is taken into account.

In the thermal modeling, some assumptions are considered: the insulator between the core and the coils is in perfect contact with both parts; there is no thermal contact between the exterior coil and the magnetic circuit; there is no thermal exchange with the air trapped between the coils and the iron; there is no convection on the upper and lower sides of the coil; there is no temperature gradient in the copper and the iron, and all surfaces have the same convection coefficient.

A magneto-thermal weak coupling is considered and the computational time is equal to 10 minutes on a single core of an Intel Xeon CPU E5-2690 at $2.60 \mathrm{GHz}$. The copper and iron losses are computed with the magnetic AC solver and introduced as heat sources in the thermal static solver. The copper temperature is used to compute the coils resistors introduced in the magnetic solver and this loop continues until change in temperatures is less than $0.1{ }^{\circ} \mathrm{C}$. Both solvers use the same mesh and are included in Opera3D software.

\section{B. Analytical Model}

In order to motivate the need of a time expensive 3D FEA model, an Analytical Model (AM) is also used to compare RBDO results with both models.

The physical phenomena within the transformer are electric, magnetic and thermal. The assumptions for AM are uniform distribution of the magnetic flux density in the iron core and no voltage drop due to the magnetizing current. The thermal assumptions are the same than the 3D FEA except that the temperatures are uniform within the coils and laminations.

The weakest points of AM are the assumption of uniform temperature in copper and iron, and the approximation of the leakage inductance values. 


\section{Optimization Problem}

The optimization problem contains 7 design variables. There are three parameters $(a, b, c)$ for the shape of the lamination, one for the frame $(d)$, two for the section of conductors $\left(S_{1}, S_{2}\right)$, and one for the number of primary turn $\left(n_{1}\right)$ (Fig. 3, left).

There are 7 inequality constraints in this problem. The copper and iron temperatures $T_{c o}, T_{i r}$ respectively should be less than $120^{\circ} \mathrm{C}$ and $100^{\circ} \mathrm{C}$. The efficiency $\eta$ should be greater than $80 \%$. The magnetizing current $I_{\mu} / I_{1}$ and drop voltage $\Delta V_{2} / V_{2}$ should be less than $10 \%$. All these constraints are computed with FEM or AM model. Finally, the filling factors of both coils $f_{1}, f_{2}$ should be lower than 0.5 .

The goal is to minimize the mass $m_{\text {tot }}$ of iron and copper materials. Thus, the optimization problem is expressed as:

$$
\begin{array}{ccc}
\min m_{\text {tot }}\left(a, b, c, d, S_{1}, S_{2}, n_{1}\right) & \text { s.t. } & \eta \geq 0.8 \\
3 \leq a \leq 30(\mathrm{~mm}) & 14 \leq b \leq 95(\mathrm{~mm}) T_{c o} \leq 120^{\circ} \mathrm{C} & I_{\mu} / I_{1} \leq 0.1 \\
6 \leq c \leq 40(\mathrm{~mm}) & 10 \leq d \leq 80(\mathrm{~mm}) T_{i r} \leq 100^{\circ} \mathrm{C} \Delta V_{2} / V_{2} \leq 0.1 \\
200 \leq n_{2} \leq 1200 & 0.15 \leq S_{1,2} \leq 19\left(\mathrm{~mm}^{2}\right) f_{2} \leq 0.5 & f_{2} \leq 0.5
\end{array}
$$

For RBDO, all constraints are considered with a target probability of failure equal to $0.13 \%$, which means a reliability index of 3. The standard deviation of each design variable is equal to $1 \%$ of its lowest bound.

\section{Results}

Table 2 shows optimal values, objective, probabilities of failure calculated by MCS with $10^{6}$ samples computed with the

\begin{tabular}{|c|c|c|c|}
\hline Values & SORA3 + FEM & SORA3 + AM & FEM reeval. \\
\hline$a$ & 12.902 & \multicolumn{2}{|c|}{13.153} \\
\hline$b$ & 46.042 & \multicolumn{2}{|c|}{51.039} \\
\hline$c$ & 18.183 & \multicolumn{2}{|c|}{16.532} \\
\hline$d$ & 42.318 & \multicolumn{2}{|c|}{43.098} \\
\hline$n_{1}$ & 659.06 & \multicolumn{2}{|c|}{641.75} \\
\hline$S_{1}$ & 0.3254 & \multicolumn{2}{|c|}{0.3216} \\
\hline$S_{2}$ & 2.7552 & \multicolumn{2}{|c|}{2.8956} \\
\hline$m_{t o t}$ & 2.4028 & 2.3552 & 2.3520 \\
\hline$P\left(T_{c o}>120^{\circ} \mathrm{C}\right)$ & $0 \%$ & $0 \%$ & $0 \%$ \\
\hline$P\left(T_{i r}>100^{\circ} \mathrm{C}\right)$ & $0.1506 \%$ & $0.1567 \%$ & $90.05 \%$ \\
\hline$P\left(\Delta V_{2} / V_{2}>0.1\right)$ & $0 \%$ & $0 \%$ & $0 \%$ \\
\hline$P\left(I_{\mu} / I_{1}>0.1\right)$ & $0.1348 \%$ & $0.1420 \%$ & $0.3281 \%$ \\
\hline$P\left(f_{1}>0.5\right)$ & $0.1327 \%$ & $0.1236 \%$ & $71.20 \%$ \\
\hline$P\left(f_{2}>0.5\right)$ & $0.1282 \%$ & $0.1307 \%$ & $0.0014 \%$ \\
\hline$P(\eta<0.8)$ & $0 \%$ & $0 \%$ & $0 \%$ \\
\hline Evaluations & 7265 & 7242 & 1 \\
\hline
\end{tabular}
meta-model, and the number of evaluations.

TABLE 2

RESULTS OF TRANSFORMER OPTIMIZATION WITH META-MODEL

For AM, SORA without meta-model is also tested and the number of evaluations is greater than 10,000, so it can be seen that SORA3 with kriging meta-model (SORA3 + AM, center column in Table 2) can find a solution almost satisfying all constraints with less evaluations. However when the same solution is reevaluated with FEM (FEM reeval., right column in Table 2), the highest probability of failure is $90 \%$, so RBDO cannot be performed with AM only. The mass computed with FEM is slightly different from the one with AM because the voltage drop is considered to calculate the number of turns for the secondary coil.

SORA3 with FEM (SORA3 + FEM, left column in Table 2) leads to a probability of failure close to its target value. The objective value is higher with FEM because AM underestimates constraints. The initial sampling includes 7,000 points evaluated in parallel on 24 cores in about 49 hours then the 265 infill sampling points are evaluated sequentially in about 44 hours. The first advantage of SORA3 with FEM is that a significant computing time can be saved as it reduces the number of evaluations. The second advantage is that kriging model gives accurate derivatives that enable the use of fast gradient-based algorithm. Contrarily, as FEM provides noisy derivatives it requires a noise-free costly algorithm when directly connected with it.

\section{CONCLUSION}

According to the mathematical example, the third strategy of kriging-based SORA is the most efficient without losing too much accuracy among the 6 approaches proposed.

RBDO of a single-phase safety isolating transformer is also performed here with FEM and the kriging-based SORA shows its applicability in dealing with this highly constrained problem by reducing the number of evaluations. Then, compared with analytical model of the same device, this approach with FEM could get a more accurate solution.

\section{REFERENCES}

[1] T. H. Lee and J. J. Jung, "A sampling technique enhancing accuracy and efficiency of metamodel-based RBDO: Constraint boundary sampling," Computers \& Structures, vol. 86, no. 13, pp. 1463-1476, 2008.

[2] B. J. Bichon, M. S. Eldred, L. P. Swiler, et al., "Efficient global reliability analysis for nonlinear implicit performance functions," AIAA journal, Vol. 46, No. 10, pp. 2459-2468, 2008.

[3] I. Lee, K. K. Choi, and L. Zhao, "Sampling-based RBDO using the stochastic sensitivity analysis and Dynamic Kriging method," Structural and Multidisciplinary Optimization, Vol. 44, No. 3, pp. 299-317, 2011.

[4] D. R. Jones, M. Schonlau, and W. J. Welch, "Efficient global optimization of expensive black-box functions," Journal of Global optimization, vol. 13, no. 4, pp. 455-492, 1998.

[5] S. Xiao, M. Rotaru, and J. K. Sykulski, "Adaptive weighted expected improvement with rewards approach in Kriging assisted electromagnetic design," IEEE Trans. Magn, vol. 49, no. 5, pp. 2057-2060, 2013.

[6] M. Schonlau. Computer Experiments and Global Optimization. $\mathrm{PhD}$ thesis, University of Waterloo, Waterloo, Canada, 1997.

[7] C. Audet, J. Denni, D. Moore, et al., "A surrogate-model-based method for constrained optimization," in 8th Symposium on Multidisciplinary Analysis and Optimization, Paper No. 4891, 2000.

[8] M. J. Sasena, Flexibility and efficiency enhancements for constrained global design optimization with kriging approximations, Ph.D. dissertation, General Motors, 2002.

[9] J. Tu, K. K. Choi, and Y. H. Park, "A new study on reliability-based design optimization," Journal of Mech. Design, vol. 121 no. 4, pp. 557564, 1999.

[10] J. Liang, Z. P. Mourelatos, and J. Tu, "A single-loop method for reliability-based design optimization," in Proceedings of ASME Design Engineering Technical Conferences, pp. 419-430, 2004.

[11] X. Du and W. Chen, "Sequential optimization and reliability assessment method for efficient probabilistic design," Journal of Mech. Design, vol. 126, no. 2, pp. 225-233, 2004.

[12] D. W. Kim, N. S. Choi, K. K. Choi, et al., "A Single-Loop Strategy for Efficient Reliability-Based Electromagnetic Design Optimization," IEEE Trans. Magn, vol. 51, no. 3, pp. 1-4, 2015.

[13] T. V. Tran, S. Brisset, and P. Brochet, "A Benchmark for Multi-Objective, Multi-Level and Combinatorial Optimizations of a Safety Isolating Transformer," in COMPUMAG, pp. 167-168, 2007. 